\title{
Sub-micrometer domain engineering on periodically poled lithium niobate
}

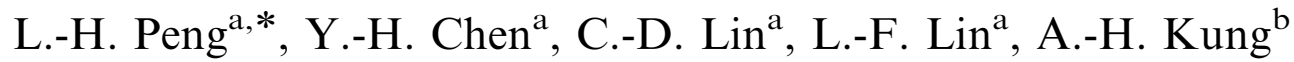 \\ ${ }^{a}$ Department of Electrical Engineering and Institute of Electro-Optical Engineering, National Taiwan University, Taipei, Taiwan \\ ${ }^{\mathrm{b}}$ Institute of Atomic and Molecular Sciences, Academia Sinica, Taipei, Taiwan
}

Available online 9 June 2006

\begin{abstract}
We report a novel method to realize high-aspect ratio $(\sim 1000)$ of submicron size inverted domains in two-dimensional periodically poled lattice structure on $0.5 \mathrm{~mm}$-thick lithium niobate $\left(\mathrm{LiNbO}_{3}\right)$ substrate. This is achieved by photolithographic patterning of $\mathrm{LiNbO}$ surface with self-organized dielectric spheres, followed by pulsed-field poling with liquid electrodes. The realization of submicron domain structures on electrically poled $\mathrm{LiNbO}_{3}$ is ascribed to a local modification of the polarization switching field due to the dielectric- and structure-induced discontinuity at the boundary between the photoresist opening and self-organized dielectric spheres.

(C) 2006 Elsevier B.V. All rights reserved.
\end{abstract}

PACS: 42.70.Mp; 77.80.Fm; 77.84.Dy; 81.65.Cf

Keywords: B1. Lithium niobate

The profound mechanical and electro-optical properties of ferroelectric crystals have made them an attractive field of study in condensed matter physics [1]. In particular, the capability for ferroelectric crystals to switch the direction of their spontaneous polarization $\left(P_{\mathrm{s}}\right)$ can result in novel device applications such as high-density data storage and nonlinear frequency conversion. The underlying physics is that the polarization direction of ferroelectric domain can be inverted by field action along the crystal's polar $(Z)$ axis and retain its stability as long as the sample is kept below the crystal's Curie temperature [2]. Ferroelectric memory devices with area density reaching $\mathrm{Tbit} / \mathrm{in}^{2}$ have recently been demonstrated on $\mathrm{LiTaO}_{3}$ film [3], $\mathrm{LiNbO}_{3}$ bulk [4] or film [5,6], and $\mathrm{RbTiOPO}_{4}[7]$ using scanning tips associated with the atomic force microscope. It is also known that polarization reversal of $P_{\mathrm{s}}$ can result in a characteristic sign change in the nonlinear susceptible tensor $\chi^{(2)}$. A periodical distribution of $180^{\circ}$ inverted domains therefore constitutes a new type of nonlinear photonic crystals which can provide a quasi-phase-matching (QPM) mechanism to compensate the material's optical dispersion and to achieve

\footnotetext{
*Corresponding author. Tel.: + 886233663649 ; fax: + 886223677467.

E-mail address: peng@cc.ee.ntu.edu.tw (L.-H. Peng).
}

efficient nonlinear wavelength conversion [8]. Recent realization of periodically poled ferroelectric crystals of lithium niobate $\left(\mathrm{LiNbO}_{3}\right)$ [9], lithium tentalate $\left(\mathrm{LiTaO}_{3}\right)$ [10], and potassium titanyl phosphate (KTP) [11] are renowned examples showing high optical gain for nonlinear parametric generation [12].

One important aspect pertinent to the ferroelectric device performance is on the control of inverted domain size and shape. Early study on the domain formation kinetics suggested a mechanism governed by the surface nucleation, fast forward domain motion, followed by sidewise domain expansion and coalescence [13]. Within the nucleation model, the lateral domain motion was ascribed to a repeated nucleation of steps along the existing $180^{\circ}$ domain walls [14]. Such phenomenon, if not controlled, can lead to irregular domain shapes that degrade the device performance. This stimulates the development of using a backswitching [15] or over-poling [16] technique to compensate the issues of domain broadening as commonly occurred to in ferroelectric switching with stripe-like electrodes due to the fringing field effect [17]. We have recently demonstrated a method of using a charged domain boundary to restrict the lateral motion of inverted domain [18] and to facilitate the formation of $2 \mathrm{D}$ periodically poled lattice structures on 
$\mathrm{LiNbO}_{3}$ [19]. To further control domain size on the patterned ferroelectric surface, conventional wisdom seeks solutions that can lead to a minimization of the crystal's internal energy at a specific lattice site. In doing so, the latter can be determined by the competition between energy stored in the crystal against that reduced by the electrostatic interaction between inverted domain and applied field [20]. Recent investigation suggests it may be possible to mitigate the polarization-switching rate by incorporating small inhomogeneities at the ferroelectricelectrode interface [21]. In this work, we report a process to achieve selective nucleation and inversion of ferroelectric domain by modifying the local switching field effect. This process consists of patterning the ferroelectric surface with photoresist and self-organized dielectric spheres [22] followed by pulsed field poling. Using this method, twodimensional nonlinear photonic crystals (2D-NPC) with domain size less than $0.5 \mu \mathrm{m}$ can be realized on $0.5 \mathrm{~mm}$ thick $\mathrm{LiNbO}_{3}$ substrate.

Our device manufacturing began with a standard photolithographic process to have a $2 \mathrm{D}$ photoresist pattern (AZ 4620, maker: www.clariant.com) developed on the $+Z$ face of congruently grown $\mathrm{LiNbO}_{3}$ substrate. A drop of mono-dispersed colloid made of polystyrene spheres (PS) and diluted in a solution of (with a 1:400 volume ratio) Triton X-100/methonal was then spun onto the surface of a photoresist patterned $\mathrm{LiNbO}_{3}$ sample. A spin speed of $4500 \mathrm{rpm}$ was found to allow a monolayer distribution of polystyrene spheres $(2.5 \mu \mathrm{m}$-dia on average $)$ to develop a self-organized, close-packed hexagonal structure when dropped into a photoresist opening $8 \mu \mathrm{m}$ wide and $4 \mu \mathrm{m}$ thick, as shown in the optical micrograph of Fig. 1. A bilayer metal contact structure of $300 \mathrm{~nm}-\mathrm{Ti} / 20 \mathrm{~nm}-\mathrm{Pt}$ was deposited onto the $+Z$ surface of the patterned $\mathrm{LiNbO}_{3}$ substrate. The sample was then transferred to a poling apparatus consisting of a Teflon cell and a pulsed voltage amplifier (Trek model 20/20A for $20 \mathrm{kV}$ and $20 \mathrm{~mA}$ output). The poling setup was similar to that originally

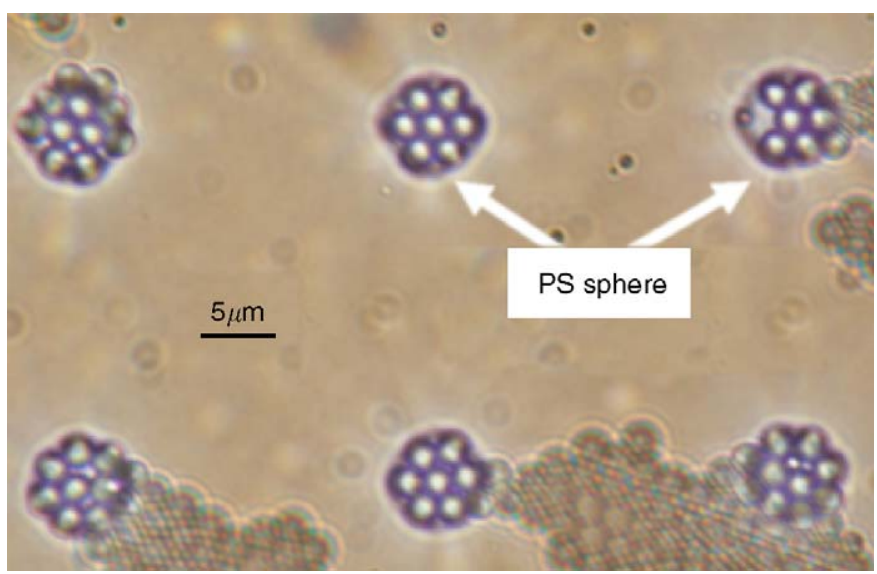

Fig. 1. Surface patterning on the $+Z$ face of $\mathrm{LiNbO}_{3}$ using photolithography with self-organized polystyrene spheres. The photo-resist opening is $\sim 8 \mu \mathrm{m}$ wide and $4 \mu \mathrm{m}$ thick with a periodicity of $29.5 \mu \mathrm{m}$. The average size of the spheres is $\sim 2.5 \mu \mathrm{m}$. designed by Myers et al. [23] in which liquid electrodes made contact with the sample surfaces via a pair of viton O-rings. To stabilize the domain reversal process, a fast turn-on rectifying diode was put in series with the poling apparatus such that relaxation of the inverted domain could be inhibited upon termination of the poling field [24]. The poled samples were then immersed in hydrofluoric acid (HF) to partially etch off the inverted domains for subsequent structure observation.

Our electrical poling procedure to form 2D periodically poled lattice structures on $\mathrm{LiNbO}_{3}$ was initiated by applying multiple electrical pulses of $1 \mathrm{~ms}$ width and $25 \mathrm{kV} / \mathrm{mm}$ magnitude to activate the polarization switching process. Here we note that use of a high-field poling pulse, with a magnitude considerably larger than the coercive field $(\sim 21 \mathrm{kV} / \mathrm{mm})$ of $\mathrm{LiNbO}_{3}$, can result in a dominant polarization switching current path to flow through the spacer regions bounded by the dielectric spheres localized at the periphery of the photoresist opening. The underlying physics is that the local polarization-switching field can be enhanced at the aforementioned lattice sites due to the dielectric- and structure-induced discontinuity. In contrast, in the central part of the photoresist opening where a smooth boundary meets at the inter-sphere spacing region, there is a smaller electric field along the crystal's polar $(Z)$ axis compared with the formal case. This would effectively introduce a nucleation rate difference and favor a selective formation of inverted domains along the periphery of the photoresist opening.

In order to prove the above analysis of discontinuity effect on the kinetics of domain formation, optical micrographs were taken from the $+Z$ and $-Z$ face of etched $\mathrm{LiNbO}_{3}$ samples that were poled with and without

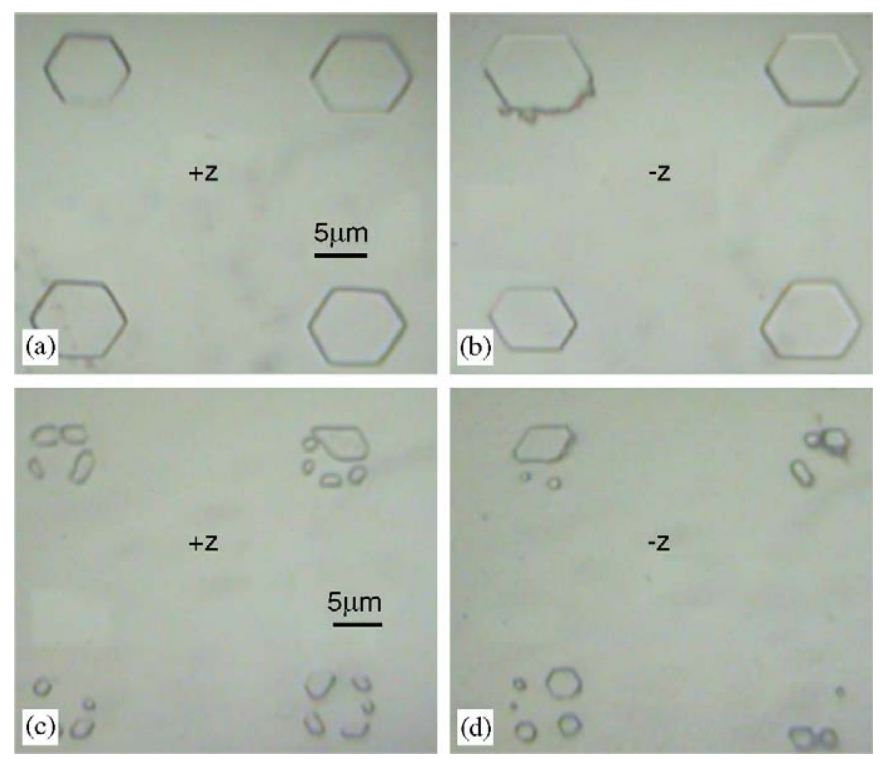

Fig. 2. Optical micrographs of inverted domain structures taken from the $+Z(\mathrm{a}, \mathrm{c})$ and $-Z(\mathrm{~b}, \mathrm{~d})$ face of etched $\mathrm{LiNbO}_{3}$ when poled with and without self-organized polystyrene spheres. The $2 \mathrm{D}$ domain periodicity is $29.5 \mu \mathrm{m}$. 

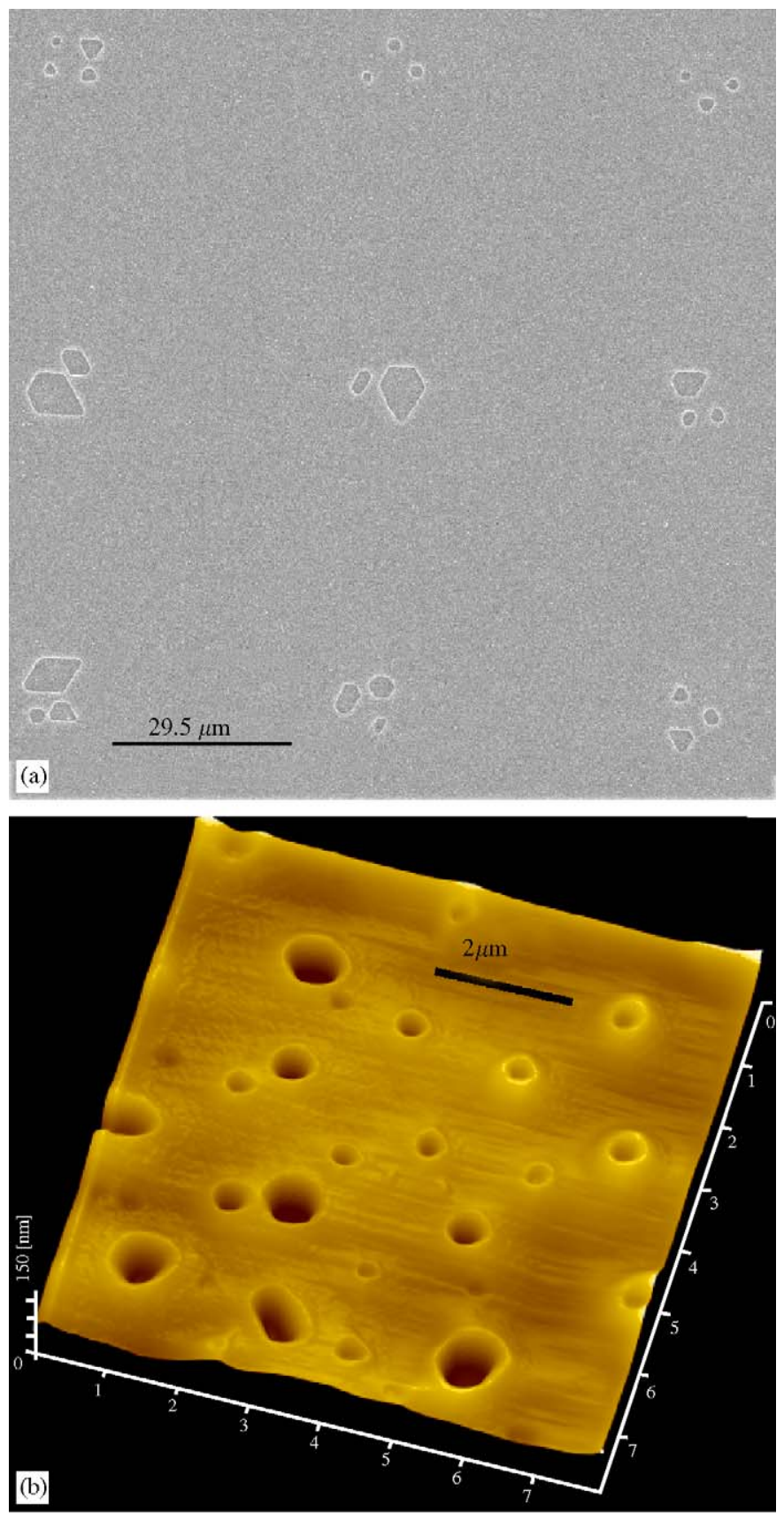

Fig. 3. (a) SEM and (b) AFM micrographs of $2 \mathrm{D}$ periodically poled devices on $0.5 \mathrm{~mm}$-thick: (a) undoped and (b) $\mathrm{ZnO}$ doped congruently grown $\mathrm{LiNbO}_{3}$ substrate.

self-organized polystyrene spheres in the photoresist opening. A close examination of the $+Z$ face image of Fig. 2(a) reveals a characteristic shape change of the inverted domain from a single, large $(\sim 5 \mu \mathrm{m})$ size of hexagon to three to five split polygons in Fig. 2(c) when self-organized polystyrene spheres were added to the photoresist opening. We further note that the corresponding $-Z$ face micrographs of Fig. 2(b) and (d) resemble a mirror image of its $+Z$ face counterpart in Figs. 2(a) and (c), respectively. This result confirms a straightforward penetration of inverted domains through the $0.5 \mathrm{~mm}$-thick $\mathrm{LiNbO}_{3}$ sub- strate when activated by the poling process outlined above. A subtle point related to device application is that an inverted ferroelectric domain of submicron size can now be conveniently made onto a thick substrate in conjunction with the electrical poling process. These observations verify our proposed model of using a photoresist pattern with self-organized dielectric spheres to selectively activate the nucleation sites of an inverted domain and to restrict its lateral motion. This process provides a novel means to create ferroelectric-based 2D nonlinear photonic crystal whose atomic form factor can be designed by the local distribution of inverted domains with self-organized dielectric spheres. Micrographs taken from a scanning electron microscope (SEM) and an atomic force microscope (AFM) on the etched $+Z$ face of periodically poled $\mathrm{LiNbO}_{3}$ devices in Fig. 3 illustrate such feasibility. Here, examples are given of 2D nonlinear photonic crystal on (a) undoped $\mathrm{LiNbO}_{3}$, and (b) $\mathrm{ZnO}$ doped $\mathrm{LiNbO}_{3}$ substrate, respectively. The corresponding $-Z$ face micrographs of these devices (not shown) exhibit mirror images to its counterpart in Fig. 3, similar to the case already shown in Fig. 2. From the domain size analysis of Fig. 2(d) or Fig. 3(b), it is found that inverted domain with a size less than $0.5 \mu \mathrm{m}$ can be conveniently made by our proposed method, suggesting it is a promising route to realize highaspect ratio $(\sim 1000)$ nonlinear photonic crystal devices.

In summary, we have demonstrated a novel device processing technique to create periodically poled domains on bulk $\mathrm{LiNbO}_{3}$ substrate. By judiciously manipulating the dielectric- and structure-induced discontinuity at the boundary between the photoresist patter and self-organized spheres, a submicron size of inverted domain structure can be realized through a $0.5 \mathrm{~mm}$-thick $\mathrm{LiNbO}_{3}$ substrate by using the conventional pulsed-field poling technique. These observations are ascribed to a unique process of selective nucleation and restrictive motion of inverted domain due to the modification of local switching field at the aforementioned lattice sites. This research was supported by National Science Council, Grants numbers NSC 93-2215-E-002-005, 94-2215-E-002-024, and 2120-M001-011.

\section{References}

[1] For a recent review, please see Ferroelectric and dielectric thin films in Handbook of Thin Film Materials, vol. 3, and Handbook of Advanced Electronic and Photonic in H.S. Nalwa (Ed.), Materials and Devices, vol. 4, Academic Press, New York, 2002 and 2001.

[2] J.F. Scott, Ferroelectric Memories, Springer, New York, 2001.

[3] Y. Hiranaga, Y. Cho, K. Fujimoto, Y. Wagatsuma, A. Onoe, Jpn J. Appl. Phys. 42 (2003) 6050.

[4] A. Agronin, Y. Rosenwaks, G. Rosenman, Appl. Phys. Lett. 85 (2004) 452.

[5] B.J. Rodriguez, R.J. Nemanich, A. Kingon, A. Gruverman, S.V. Kalinin, K. Terabe, X.Y. Liu, K. Kitamura, Appl. Phys. Lett. 86 (2005) 012906.

[6] K. Terabe, M. Nakamura, S. Takekawa, K. Kitamura, S. Higuchi, Y. Gotoh, Y. Cho, Appl. Phys. Lett. 82 (2003) 433. 
[7] Y. Rosenwaks, D. Dahan, M. Molotskii, G. Rosenman, Appl. Phys Lett. 86 (2005) 012909.

[8] J.A. Armstrong, N. Bloembergen, J. Ducuing, P.S. Pershan, Phys. Rev. 127 (1962) 1918.

[9] Y. Yamada, N. Nada, M. Saitoh, K. Watanabe, Appl. Phys. Lett. 62 (1993) 435.

[10] K. Mizuuchi, K. Yamamoto, M. Kato, Appl. Phys. Lett. 70 (1997) 1201.

[11] H. Karlsson, F. Laurell, L.K. Cheng, Appl. Phys. Lett. 74 (1999) 1519.

[12] L.E. Myers, R.C. Eckardt, M.M. Fejer, R.L. Byer, W.R. Bosenberg, J.W. Pierce, J. Opt. Soc. Am. B 12 (1995) 2102.

[13] E. Fatuzzo, W.J. Merz, Phys. Rev. 116 (1959) 61.

[14] R.C. Miller, G. Weinreich, Phys. Rev. 117 (1960) 1460.

[15] R.G. Batchko, V.Y. Shur, M.M. Fejer, R.L. Byer, Appl. Phys. Lett. 75 (1999) 1673.
[16] A.C. Busacca, C.L. Sones, V. Apostolopoulos, R.W. Eason, S. Mailis, Appl. Phys. Lett. 81 (2002) 4946.

[17] G. Rosenman, Kh. Garb, A. Skliar, M. Oron, D. Eger, M. Katz, Appl. Phys. Lett. 73 (1998) 8651.

[18] L.-H. Peng, Y.-J. Shih, Y.-C. Zhang, Appl. Phys. Lett. 81 (2002) 1666.

[19] L.-H. Peng, Y.-C. Shih, S.-M. Tsang, C.-C. Hsu, Appl. Phys. Lett. 81 (2002) 5210.

[20] R. Landauer, J. Appl. Phys. 28 (1957) 227.

[21] G. Gerra, A.K. Tagantsev, N. Setter, Phys. Rev. Lett. 94 (2005) 107602.

[22] J.C. Hulteen, R.P. Van Duyne, J. Vac. Sci. Technol. A 13 (1995) 1553.

[23] L.E. Myers, R.C. Eckardt, M.M. Fejer, R.L. Byer, W.R. Bosenberg, J.W. Pierce, J. Opt. Soc. Am. B 12 (1995) 2102.

[24] L.-H. Peng, Y.-C. Zhang, Y.-C. Lin, Appl. Phys. Lett. 78 (2001) 4. 